\title{
Relationship of AM to PM Noise in Selected RF Oscillators
}

\author{
Lisa M. Nelson, Craig W. Nelson, and Fred L. Walls, Senior Member, IEEE
}

\begin{abstract}
We have studied the amplitude modulation (AM) and phase modulation (PM) noise in a number of $5 \mathrm{MHz}$ and $100 \mathrm{MHz}$ oscillators to provide a basis for developing models of the origin of $A M$ noise. To adequately characterize the $A M$ noise in high performance quartz oscillators, we found it necessary to use two-channel cross-correlation AM detection. In the quartz oscillators studied, the power spectral density (PSD) of the $f^{-1}$ and $f^{0}$ regions of AM noise is closely related to that of the PM noise. The major difference between different oscillators of the same design depends on the flicker noise performance of the resonator. We therefore propose that the $f^{-1}$ and $f^{0}$ regions of $A M$ and $P M$ noise arise from the same physical processes, probably originating in the sustaining amplifier.
\end{abstract}

\section{INTRODUCTION}

T IO OUR KNOWLEDGE there has been little quantitative work analyzing the relationship between $\mathrm{AM}$ and $\mathrm{PM}$ noise in oscillators. Most of the literature assumes that the AM noise is much smaller than the PM noise. Leeson, Parker, and others have described models of PM noise in oscillators [1]-[3]. We are not aware, however, of any theory for the spectral dependence of the AM noise close to the carrier. We have therefore undertaken a detailed study of PM and AM noise in several quartz oscillators at 5 and $100 \mathrm{MHz}$ to provide a basis for developing models of the origin of AM noise.

To adequately characterize the AM noise in high performance quartz oscillators, we found it necessary to use two-channel cross-correlation AM detection. The signal is split by a reactive power splitter into two signals that are each AM detected. The resulting baseband signals are then amplified and the power spectral density (PSD) of the cross spectrum between the two channels is computed. This technique provides a noise floor of order $-190 \mathrm{dBc} / \mathrm{Hz}$ for $\mathrm{AM}$ noise at 50 $\mathrm{kHz}$ and $-160 \mathrm{dBc} / \mathrm{Hz}$ at $10 \mathrm{~Hz}$ for signals of approximately $+13 \mathrm{dBm}$.

An improved three-cornered-hat technique using crosscorrelation makes PM noise measurements more quickly and accurately than the traditional three-cornered-hat measurement technique [4], [5]. In this approach the noise contribution of both the two references and the measurement systems average down to zero as $\frac{1}{\sqrt{N}}$ where $N$ is the number of measurnments taken. In our setup we were able to obtain a PM noise floor

\footnotetext{
Manuscript received October 25, 1993; revised April 11, 1994; accepted April 13, 1994. This work was supported in part by the Calibration Coordination Group of JCTG/GMT-JLC and the Space and Terrestrial Communications Evaluation Division. Tactical Support Branch, Ft. Monmouth, NJ.

The authors are with the Time and Frequency Division, National Institute of Standards and Technology, Boulder, CO 80303 USA.

IEEE Log Number 9403344
}

of $1 / 2 S_{\phi}(f)=-160 \mathrm{dBc} / \mathrm{Hz}$ at $10 \mathrm{~Hz}$ and $1 / 2 S_{\phi}(f)=-190$ $\mathrm{dBc} / \mathrm{Hz}$ at $50 \mathrm{kHz}$.

In many of the quartz oscillators studied, the PSD of the $f^{-1}$ and $f^{0}$ regions of AM noise is closely related to that of the PM noise over a substantial range in Fourier frequency. The major difference between different oscillators of the same design depends on the flicker performance of the resonator. $\mathrm{AM}$ and PM noise generally differ only in the region where the PM noise varies as $f^{-3}$ (flicker FM noise) or in some cases $f^{-4}$ (random walk FM noise). For example, we have found that the AM noise of $5 \mathrm{MHz}$ oscillators is quite similar to the PM noise for Fourier frequencies above approximately $3 \mathrm{~Hz}$. Likewise, the AM and PM noise of the $100 \mathrm{MHz}$ quartz oscillators studied were quite similar for Fourier frequencies above approximately $300 \mathrm{~Hz}$.

The relationship between the AM and PM noise can be exploited in many areas of analysis to assist in the design of new oscillators. It is tedious to determine the PM performance of a new oscillator design, especially one that exhibits performance superior to existing references. At the very minimum three oscillators of similar performance are required. (The requirements on the performance of the other oscillators can be relaxed considerably if the modified threecornered-hat approach is used [4], [5].) On the other hand the AM performance of a single superior oscillator can be more easily assessed using the two-channel cross-correlation AM detection techniques presented in this paper. Therefore, it can be beneficial to use the readily obtained AM performance to evaluate the noise more quickly.

\section{Description of AM Detection Circuits}

Fig. 1 shows the single-sideband noise floor for a variety of available $\mathrm{AM}$ detectors at $5 \mathrm{MHz}$. All exhibit noise floors or resolution far above the phase noise of available oscillators at Fourier frequencies above $1 \mathrm{kHz}$ and hence are unsuitable for measurements of the AM noise in these oscillators. Montress et al., have used double-balanced mixers as phase detectors with good results at drive powers of $+23 \mathrm{dBm}$ [6]. Typically the oscillators with the best phase noise performance at 5 and $100 \mathrm{MHz}$ have outputs of $+13 \mathrm{dBm}$. Typical double-balanced mixers operated at this power level have relatively high levels of noise (see Fig. 2). The problem has been overcome by combining the approach of Montress et al. with a two-channel cross-correlation technique [4]-[8]. Fig. 3 shows the full AM measurement system schematic that was used for the crosscorrelation AM noise detection. 


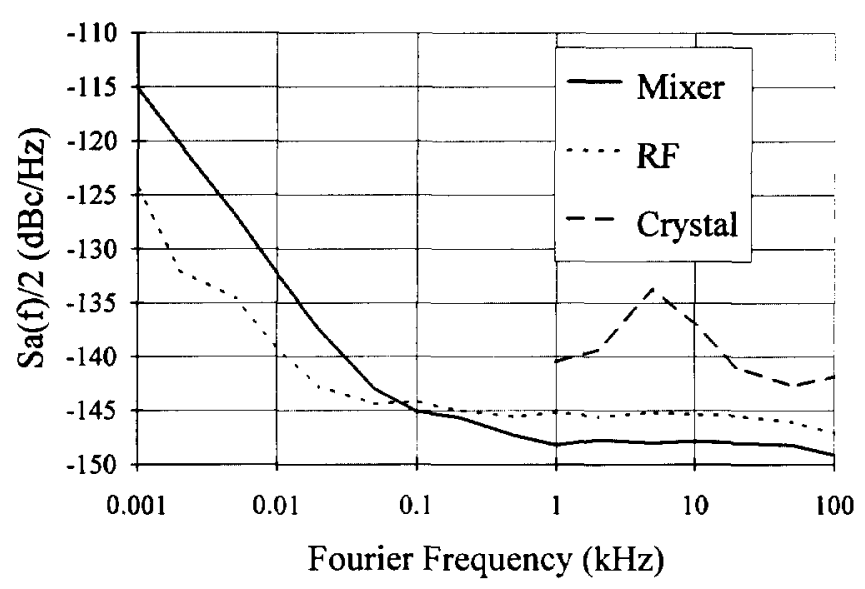

Fig. 1. AM noise floor of various detectors at 5 and $100 \mathrm{MHz}$ at $13 \mathrm{dBm}$. The noise of a mixer is included for comparison. The mixer is a WJ M9HC, the RF detector is a WBE model A33, and the Crystal Detector is a HP 8472B.

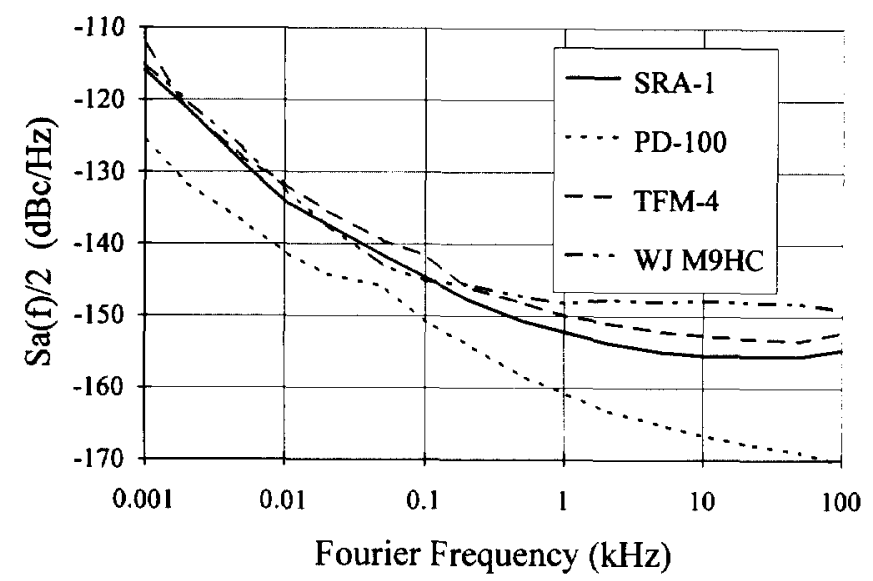

Fig. 2. AM noise floors of two mixer types at 5 and $100 \mathrm{MHz}$ at $13 \mathrm{dBm}$. The PD-100 is a single-balanced mixer, the SRA-1 is a double-balanced mixer, the TFM-4 is a double-balanced mixer,and the WJ M9HC is a double-balanced mixer.

\section{Measurement Techniques}

At the output of the spectrum analyzer we obtain $S_{a}(f) / 2$ for AM noise, or $S_{\phi}(f) / 2$ for PM noise measurements. These are the single-sideband spectral densities of our signal. The practical definition for the power spectral density of amplitude fluctuations is given by

$$
S_{a}(f)=\frac{[\Delta \varepsilon(f)]^{2}}{V_{0}^{2}} \frac{1}{\mathrm{BW}}
$$

where $\Delta \varepsilon(f)$ is the change in amplitude measured at Fourier separation $f$ from the carrier, $V_{0}$ is the average carrier voltage and $\mathrm{BW}$ is the noise bandwidth of the spectrum analyzer. For the measurement system shown in Fig. 3, (1) becomes

$$
S_{a}(f)=\frac{\operatorname{PSD}\left(V_{n}\right)}{\left[k_{a} G(f)\right]^{2}}
$$

where PSD denotes power spectral density, $V_{n}$ is the noise voltage out, $k_{a}$ is the sensitivity factor of the detector for converting $\Delta \varepsilon / V_{0}$ to a voltage, and $G(f)$ is the amplifier gain versus Fourier frequency.

At the output of the spectrum analyzer we obtain the power spectral density (PSD) for each of the two channels.

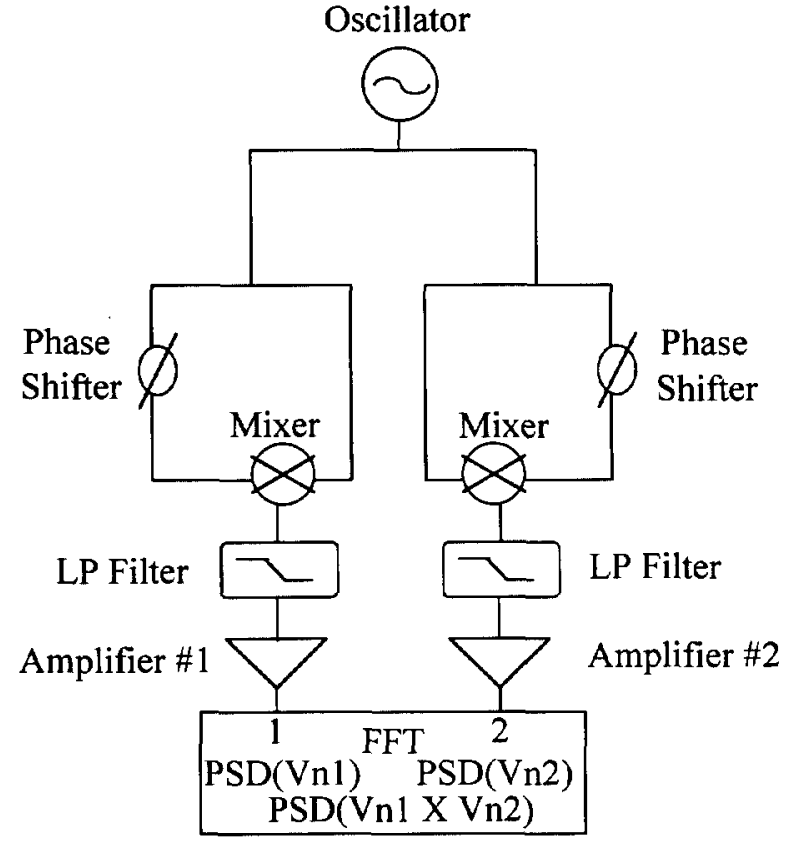

Fig. 3. AM cross-correlation measurement system.

This provides us with the noise from the source and the measurement noise in each respective channel [see (3) and (4)]. By using cross-correlation we can obtain the AM noise of the source as shown in (5). The uncorrelated noise between the channels averages away as the reciprocal of the square root of the number of averages.

$$
\begin{aligned}
\operatorname{PSD}\left(V_{n 1}\right)= & \alpha\left(\mathrm{AM}_{\text {Source }}+\text { System }_{1}\right), \\
\operatorname{PSD}\left(V_{n 2}\right)= & \alpha\left(\mathrm{AM}_{\text {Source }}+\text { System }_{2}\right), \\
\operatorname{PSD}\left(V_{n 1} \times V_{n 2}\right)= & \alpha\left[\mathrm{AM}_{\text {Source }}\right. \\
& \left.\times\left(+\frac{\text { System }_{1}+\text { System }_{2}}{N^{1 / 2}}\right)\right],
\end{aligned}
$$

$$
\begin{aligned}
& \operatorname{PSD}\left(V_{n 1}\right)-\operatorname{PSD}\left(V_{n 1} \times V_{n 2}\right)=\alpha\left(\text { System }_{1}\right), \\
& \operatorname{PSD}\left(V_{n 2}\right)-\operatorname{PSD}\left(V_{n 1} \times V_{n 2}\right)=\alpha\left(\text { System }_{2}\right),
\end{aligned}
$$

where the proportionality constant $\alpha=\left[k_{a} G(f)\right]^{2}$. Subtracting the source noise from (3) and (4), respectively, we are able to obtain the noise in each channel [see (6) and (7)].

\section{AM AND PM NOISE FOR SElEcted OSCILlators}

AM and PM noise measurements on various oscillators are described below. The mathematical models for their relationships are presented in the following section.

Fig. 4 shows the AM and PM noise of a $5 \mathrm{MHz}$ oscillator that uses an AT-cut resonator driven at low power. The AM noise was measured using the techniques described in the previous section. Calibration of the PM noise was done using a three-cornered-hat technique outlined in [8]. The PM noise in the 0.3 to $3 \mathrm{~Hz}$ range shows flicker frequency $\left(f^{-3}\right)$ as expected with white PM noise at higher Fourier frequencies. 


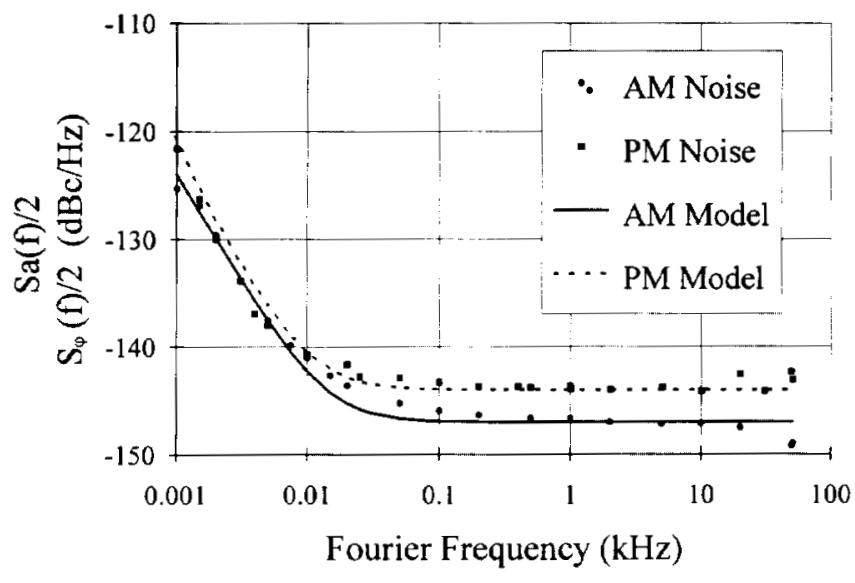

Fig. 4. AM and PM noise with models for AT-cut $5 \mathrm{MHz}$ oscillator.

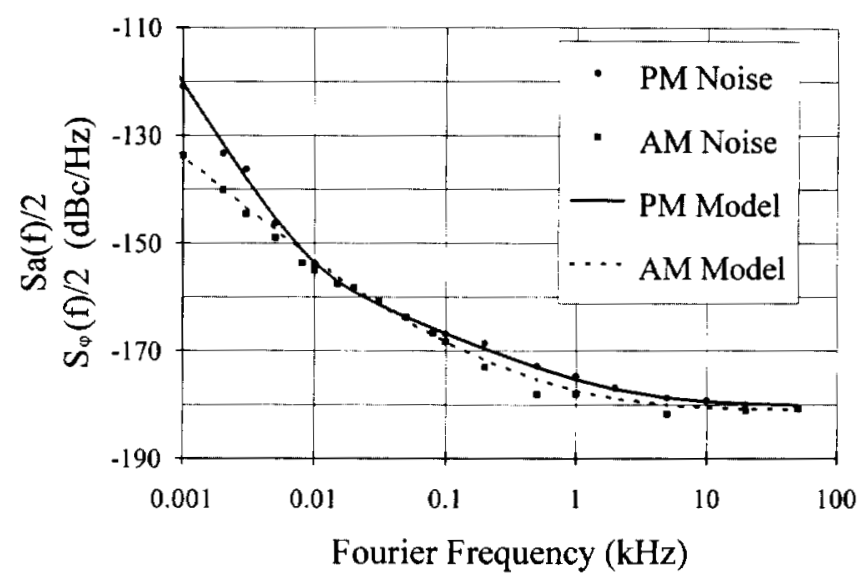

Fig. 5. AM and PM noise with models for SC-cut $5 \mathrm{MHz}$ oscillator.

The AM noise shows $f^{-2}$ (white frequency) behavior as does the PM noise until $30 \mathrm{~Hz}$ and continues to follow the white PM noise to higher Fourier frequencies. The models for the AM and PM noise are both similar for the $f^{-2}$ and $f^{0}$ components of the noise.

Fig. 5 shows $\mathrm{AM}$ and $\mathrm{PM}$ noise of a $5 \mathrm{MHz}$ high performance oscillator that uses an SC-cut resonator driven at relatively high power. The AM and PM noise were measured with the techniques described above. The PM noise exhibits flicker FM, flicker PM, and white PM noise. The AM noise tracks the PM noise from approximately $10 \mathrm{~Hz}$ to at least 50 $\mathrm{kHz}$. The models for the AM and PM noise are both similar for $f^{-1}$ and $f^{0}$ components of the noise.

Fig. 6 shows $\mathrm{AM}$ and $\mathrm{PM}$ noise of another $5 \mathrm{MHz}$ high performance oscillator of the same SC-cut design as Fig. 5. The PM noise was measured using a cross-correlation technique described by Walls [5]. Again the PM noise exhibits flicker FM, flicker PM, and white PM noise. The AM noise close to the carrier has a white frequency $\left(f^{-2}\right)$ component but follows the PM noise in both the flicker PM and white PM noise characteristics at higher frequencies.

Fig. 7 shows AM and PM noise of a high performance 100 $\mathrm{MHz}$ oscillator. The AM noise was measured with the technique previously described. A three-cornered-hat technique was used to obtain both the AM and PM noise. The PM noise

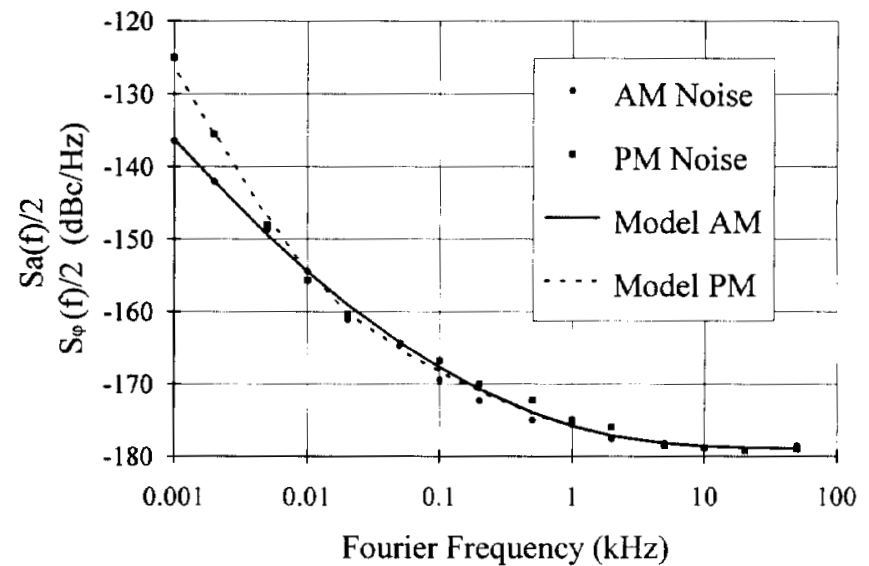

Fig. 6. $\mathrm{AM}$ and $\mathrm{PM}$ noise with models for $\mathrm{SC}$-cut $5 \mathrm{MHz}$ oscillator.

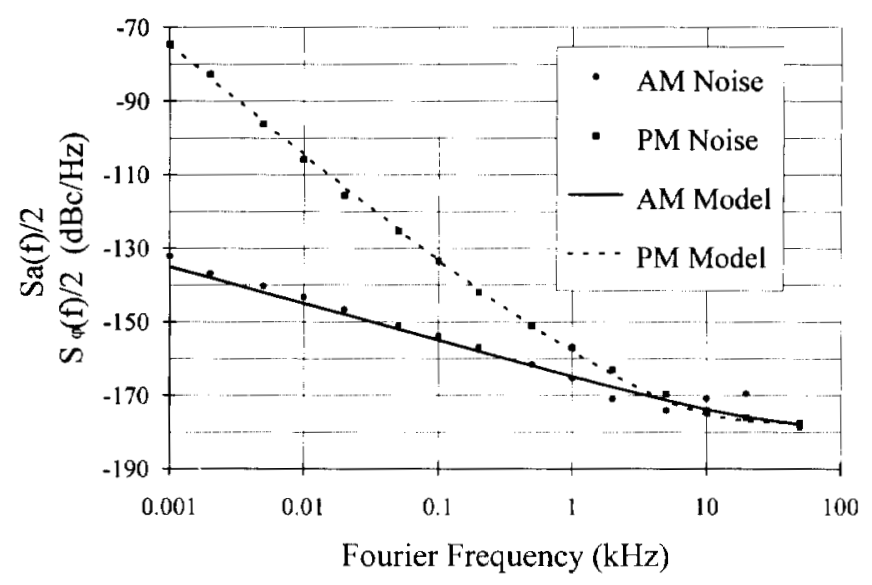

Fig. 7. $\mathrm{AM}$ and $\mathrm{PM}$ noise with models for SC-cut $100 \mathrm{MHz}$ oscillator.

fits the classic model described by Parker with flicker FM, white frequency, and white PM noise [10]. The AM noise falls as $f^{-1}$ and joins with the PM noise at higher frequencies.

Fig. 8 shows AM and PM noise for another high performance $100 \mathrm{MHz}$ oscillator. The AM noise was measured with the same technique as described above. The PM noise was measured using the modified three-cornered-hat technique outlined in [4]. The PM noise follows Parker's model with flicker FM, white frequency, and white PM noise [10]. The AM noise falls as $f^{-1}$ and follows the white PM noise of the PM noise at higher frequencies from the carrier.

\section{DISCUSSION}

The AT-cut $5 \mathrm{MHz}$ oscillator, shown in Fig. 4, fits the following models with minimal variations:

$$
\begin{aligned}
& \text { PM Model: } \frac{10^{-12.5}}{f^{4}}+\frac{10^{-13.9}}{f^{3}}+\frac{10^{-12.3}}{f^{2}}+10^{-14.4} \\
& \text { AM Model: } \frac{10^{-12.4}}{f^{2}}+10^{-14.7}
\end{aligned}
$$

The AM and PM models differ by $1 \mathrm{~dB}$ for the $f^{-2}$ noise and in the $f^{0}$ component of the noise by $3 \mathrm{~dB}$. The SC-cut $5 \mathrm{MHz}$ 


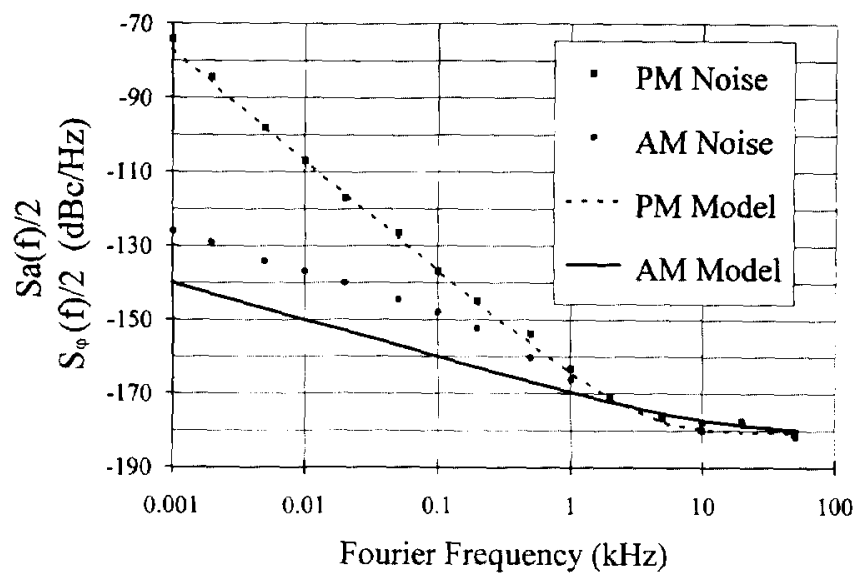

Fig. 8. AM and PM noise with models for SC-cut $100 \mathrm{MHz}$ oscillator.

oscillator in Fig. 5 follows the following models:

$$
\begin{aligned}
& \text { PM Model: } \frac{10^{-12.1}}{f^{4}}+\frac{10^{-12.8}}{f^{3}}+\frac{10^{-14.7}}{f}+10^{-18.02} . \\
& \text { AM Model: } \frac{10^{-13.4}}{f^{2}}+\frac{10^{-15}}{f}+10^{-18.1}
\end{aligned}
$$

The $f^{-1}$ noise of the AM and PM models in this case differ by $3 \mathrm{~dB}$ and the $f^{0}$ component by only eight tenths of a $\mathrm{dB}$. The $5 \mathrm{MHz}$ oscillator in Fig. 6 shows a similar AM and PM model relationship as the previous $\mathrm{SC}$-cut $5 \mathrm{MHz}$ oscillator with only slight variations in the exponents of the model:

$$
\begin{array}{ll}
\text { PM Model: } & \frac{10^{-12.65}}{f^{3}}+\frac{10^{-14.87}}{f}+10^{-17.9} \\
\text { AM Model: } & \frac{10^{-13.65}}{f^{2}}+\frac{10^{-14.87}}{f}+10^{-17.9}
\end{array}
$$

An interesting feature in these AM and PM models is that the $f^{-1}$ and $f^{0}$ noise components are identical.

The $100 \mathrm{MHz}$ oscillator in Fig. 7 resembles the models from Fig. 8 quite closely except for a slight difference in the $f^{-2}$ component of the PM noise model. The similarities between the AM and PM models of this oscillator are seen in the $f^{0}$ component where there is approximately a $3 \mathrm{~dB}$ difference between the two models:

$$
\begin{aligned}
& \text { PM Model: } \frac{10^{-7.46}}{f^{3}}+\frac{10^{-9.9}}{f^{2}}+10^{-17.77} . \\
& \text { AM Model: } \frac{10^{-13.5}}{f}+10^{-18} .
\end{aligned}
$$

The $100 \mathrm{MHz}$ oscillator in Fig. 8 matches the following models with slight discrepancies between the model and the close in data. In this case the $f^{0}$ noise characteristics are identical:

$$
\begin{aligned}
& \text { PM Model: } \frac{10^{-7.7}}{f^{3}}+\frac{10^{-10.8}}{f^{2}}+10^{-18.06} . \\
& \text { AM Model: } \frac{10^{-14}}{f}+10^{-18.06}
\end{aligned}
$$

We do not understand the differences between the models for our $100 \mathrm{MHz}$ AM and PM noise measurements. However, the model characteristics of Fig.s 7 and 8, whose oscillators were of similar cut and brand, have similar AM and PM models for their $f^{0}$ component. The $f^{0}$ model differs at most by $3 \mathrm{~dB}$. The $f^{-2}$ component, however, differs by about $9 \mathrm{~dB}$.

\section{CONCLUSION}

The model relationships between the various $5 \mathrm{MHz}$ oscillators present strong evidence to suggest that there is a connection between the AM and PM noise for a specific oscillator. $f^{-1}$ and $f^{0}$ components in both the AM and PM noise seem to come from the same source. The $100 \mathrm{MHz}$ oscillator relationships are inconclusive in this respect, although they maintain similar model relationships between the two SC-cut oscillators tested. We speculate that the difference in the model and the data in Fig. 8 could be due to compression in the output amplifier of our system. The $f^{0}$ component of the AM and PM noise for the $100 \mathrm{MHz}$ oscillators, however, seems to be similar and should give some information about the thermal noise characteristics in our oscillators.

\section{ACKNOWLEDGMENT}

The authors thank H. D. Ascarrunz, E. S. Ferre, and F. G. Ascarrunz for their help with the measurements.

\section{REFERENCES}

[1] D. B. Leeson, "A simple model of feedback oscillator noise spectrum," Proc. IEEE, vol. 54, no. 2, pp. 329-330, Feb. 1966.

[2] T. E. Parker and G. K. Montress, "Spectral purity of acoustic resonator oscillators," Proc. 1992 IEEE Freq. Contr. Symp., 1992, pp. 340-348.

[3] G. S. Curtis, "The relationship between resonator and oscillator noise, and resonator noise measurement techniques," Proc. 4lst Ann. Symp. on Freq. Contr., 1987, pp. 420-428.

[4] F. L. Walls, "Reducing errors, complexity, and measurement time for pm noise measurements," Proc. 1993 IEEEInt. Freq. Contr. Symp., 1993, pp. 289-297.

[5] W. F. Walls, "Cross-correlation phase noise measurements," Proc. 1992 IEEE Freq. Contr. Symp., 1992, pp. 257-261.

[6] G. K. Montress, T. E. Parker, and M. J. Loboda, "Residual phase noise measurements of VHF, UHF and microwave components," Proc. $43 r d$ Ann. Symp. Freq. Contr., 1989, pp. 349-359.

[7] A. L. Lance, W. D. Seal, and F. Labaar, Infrared and Millimeter Waves. San Diego, CA: Academic, Vol. II, 1984, ch. 7, pp. 284-286.

[8] F. L. Walls, A. J. D. Clements, C. M. Felton, and T. D. Martin "Precision phase noise metrology," Proc. Nat. Conf. Standards Lat. (NCSL), 1991, pp. 257-275.

[9] Commercial equipment is identified in this paper in order to specify adequately the experimental design. This identification does not imply recommendation or endorsement by NIST, nor does it imply that the equipment is the best available for the purpose.

[10] T. E. Parker, "Characteristics and sources of phase noise in stable oscillators," Proc. 41st Ann. Symp. on Freq. Contr., 1987, pp. 99-110

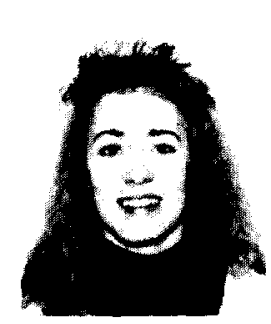

Lisa M. Nelson was born in Denver, CO on May 20,1972 . She is an undergraduate working toward a B.S. degree in electrical engineering from the University of Colorado, Boulder.

Since 1991, she has been a student research assistant in the Time and Frequency Division at the National Institute of Standards and Technology, Boulder, CO. She is presently working in the phase noise metrology group. Her research is in lownoise frequency stability measurement systems and accurate phase noise metrology. She has published

four papers.

Ms. Nelson is a member of the Society of Women Engineers, Tau Beta Pi. and Eta Kappa Nu. 


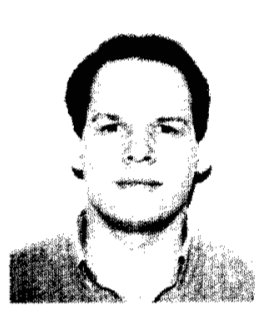

Craig W. Nelson was born in Washington, DC on February 26, 1967. After growing up in West Germany, he received the Bachelor degree in electrical engineering from the University of Colorado, Boulder in 1990.

As an undergraduate working for NIST, he was involved in the design of the electronics package for NIST-7, the optically pumped cesium standard. He also worked for Bernoulli Optical Systems, developing a write-once-read-many optical drive using flexible optical media. Since graduating he has been working as a consultant for NIST, specializing in ultralow phase noise synthesis and metrology in the RF and microwave range. $\mathrm{He}$ has published three scientific papers.

Mr. Nelson is an EIT.
Fred L. Walls (SM'94) for a photograph and biography, see p. 521 of the July 1994 issue of this TRANSACTIONS 